\title{
ABSTRACT FROM CURRENT LITERATURE
}

Cardiovascular and Renal Outcomes with Empagliflozin in Heart Failure

Packer M, Anker SD, Butler J, et al

$N$ Engl J Med 2020; 383: 1413-24

DOI: $10.1056 /$ NEJMoa2022190

Background: Sodium glucose cotransporter 2 (SGLT2) inhibitors reduce the risk of hospitalization for heart failure in patients regardless of the presence or absence of diabetes. More evidence is needed regarding the effects of these drugs in patients across the broad spectrum of heart failure, including those with a markedly reduced ejection fraction.

Methods : In this double blind trial, we randomly assigned 3730 patients with class II, III or IV heart failure and an ejection fraction of $40 \%$ or less to receive empagliflozin (10 $\mathrm{mg}$ once daily) or placebo, in addition to recommended therapy. The primary outcome was a composite of cardiovascular death or hospitalization for worsening heart failure.

Results: During a median of 16 months, a primary outcome event occurred in 361 of 1863 patients $(19.4 \%)$ in the empagliflozin group and in 462 of 1867 patients $(24.7 \%)$ in the placebo group (hazard ratio for cardiovascular death or hospitalization for heart failure, 0.75; 95\% confidence interval [CI], 0.65 to $0.86 ; \mathrm{P}<0.001)$. The effect of empagliflozin on the primary outcome was consistent in patients regardless of the presence or absence of diabetes. The total number of hospitalizations for heart failure was lower in the empagliflozin group than in the placebo group (hazard ratio, $0.70 ; 95 \% \mathrm{CI}, 0.58$ to 0.85 ; $\mathrm{P}<0.001)$. The annual rate of decline in the estimated glomerular filtration rate was slower in the empagliflozin group than in the placebo group ( 0.55 vs. $2.28 \mathrm{ml}$ per minute per $1.73 \mathrm{~m} 2$ of body surface area per year, $\mathrm{P}<0.001)$, and empagliflozin-treated patients had a lower risk of serious renal outcomes. Uncomplicated genital tract infection was reported more frequently with empagliflozin.

Conclusions: Among patients receiving recommended therapy for heart failure, those in the empagliflozin group had a lower risk of cardiovascular death or hospitalization for heart failure than those in the placebo group, regardless of the presence or absence of diabetes. (Funded by Boehringer Ingeyheim and Eli Lilly; EMPERORReduced Clinical Trials gov number, NCT03057977).
Long term cardiovascular safety of febuxostat compared with allopurinol in patients with gout (FAST): a multicentre, prospective, randomised, open-label, non-inferiority trial

Mackenzie IS, Fold I, Nuki G, et al

Lancet 2020; 396: 1745-57

DOI: org/10.1016/S0140-6736

Background: Febuxostat and allopurinol are urate-lowering therapies used to treat patients with gout. Following concerns about the cardiovascular safety of febuxostat, the European Medicines Agency recommended a post licensing study assessing the cardiovascular safety of febuxostat compared with allopurinol.

Methods: We did a prospective, randomised, openlebel, blinded endpoint, non inferiority trial of febuxostat versus allopurinal in patients with gout in the UK, Denmark and Sweden. Eligible patients were 60 years or older already receiving allopurinal and had at least one additional cardiovascular risk factor. Those who had myocardial infarction or stroke in the previous 6 months or who had severe congestive heart failure or severe renal impairment were excluded. After a lead-in phase in which allopurinol dose was optimised towards achieving a serum urate concentration of less than $0.357 \mathrm{mmol} / \mathrm{L} \quad(<6$ $\mathrm{mg} / \mathrm{dL})$, patients were randomly assigned $(1: 1$, with stratification according to previous cardiovascular events) to continue allopurinal (at the optimised dose) or start febuxostat at 80 $\mathrm{mg} /$ day, increasing to $120 \mathrm{mg} /$ day if necessary to achieve the target serum urate concentration. The primary outcome was a composite of hospitalisation for non-fatal myocardial infarction or biomaker-positive acute coronary syndrome; non fatal stroke; or cardiovascular death. The hazard ratio (HR) for febuxostat versus allopurinal in a Cox proportional hazards model (adjusted for the stratification variable and country) was assessed for non-inferiority (HR limit 1.3) in an on-treatment analysis. This study is registered with the EU Clinical Trials Register (EudraCr 2011- 001883-23) and ISRCTN (ISRCTN72443728) and is now closed.

Findings From Dec 20, 2011, to Jan 26, 2018, 6128 patients (mean age 710 years [SD 6.4] 5225 [85.3\%] men 903 [14.7\%] women, 2046 [33.4\%] with previous cardiovasculair disease) were enrolled and randomly allocated to receive allopurinal $(n=3065)$ or febuxostat $(n=3063)$. By the study end date (Dec 31, 2019), 189 (6.2\%) patients in the febuxostat group and 169 (5.5\%) in the allopurinol group withdrew from all follow up. Median follow up time was 1467 days (IQR 10292052) and median on-treatment follow up was 1324 days (IQR 870-1919). For incidence of the primary endpoint, on treatment, 
febuxostat (172 patients [1.72 events per 100 patient years]) was non inferior to allopurinol (241 patients [2.05 events per 100 patient years], adjusted HR 0.85 [95\% CI 0.70-1.03], p<0.0001). In the febuxostat group, $222(7.2 \%)$ of 3063 patients died and $1720(57.3 \%)$ of 3001 in the safety analysis set had at least one serious adverse event (with 23 events in 19 [0.6\%] patients related to treatment). In the allopurinal group, $263(8.6 \%)$ of 3065 patients died and $1812(59.4 \%)$ of 3050 had one or more serious adverse events (with five events in five [0.2\%] patients related to treatment). Randomised therapy was discontinued in $973(32.4 \%)$ patients in the febuxostat group and 503 (16.5\%) patients in the allopurinol group.

Interpretation: Febuxostat is non-inferior to allopurinol therapy with respect to the primary cardiovascular endpoint, and its long-term use is not with an increased risk of death or serious adverse events compared with allopurinol.

Red meat intake and risk of coronary heart disease among US men: prospective cohort study Al-Shaar L, Satija, Wang DD, et al

BMJ 2020; 371: $m 41411$

doi: 10.1136 bmjm 4141

Objectives: To study total, processed, and unprocessed red meat in relation to risk of coronary heart disease (CHD) and to estimate the effects of substituting other protein sources for red meat with CHD risk.

Design: Prospective cohort study with repeated measures of diet and lifestyle factors.

Setting: Health Professionals Follow up Study cohort, United States, 1986-2016.

Participants: 43,272 men without cardiovascular disease or cancer at baseline

Main Outcome Measures: The primary outcome was total CHD, comprised of acute non-fatal myocardial infarction or fatal CHD. Cox models were used to estimate hazard ratios and 95\% confidence intervals across categories of red meat consumption. Substitution analyses were conducted by comparing coefficients for red meat and the alternative food in models, including red meat and alternative foods as continuous variables.

Results: During 1,023,872 person years of follow up, 4456 incident CHD events were documented of which 1860 were fatal. After multivariate adjustment for dietary and non-dietary risk factors, total, unprocessed, and processed red meat intake were each associated with a modestly higher risk of CHD (hazard ratio for one serving per day increment: 1.12 (95\% confidence interval 1.06 to 1.18 ) for total red meat. 1.11 (1.02 to 1.21$)$ for unprocessed red meat and 1.15 (1.06 to 1.25) for processed red meat).
Compared with red meat, the intake of one serving per day of combined plant protein sources (nuts, legumes, and soy) was associated with a lower risk of CHD (0.86 (0.80 to 0.93) compared with total red meat. 0.87 (0.79 to 0.95), compared with unprocessed red meat and 0.83 (0.76 to 0.91) compared with processed red meat). Substitutions of whole grains and dairy products for total red meat and eggs for processed red meat were also associated with lower CHD risk.

Conclusions: Substituting high quality plant foods such as legumes, nuts, or soy for red meat might reduce the risk of CHD. Substituting whole grains and dairy products for total red meat and eggs for processed red meat, might also reduce this risk.

\section{Safety of ceftriaxone in paediatrics: a systematic review}

Zeng L, Wang $C$, Jiang $M$, et al

Arch Dis Child 2020; 105: 981-985

Doi: 10.1136/archdischild-2019-317950

Objective: To determine the safety of ceftriaxone in paediatric patients and systematically evaluate the categories and incidences of adverse drug reactions (ADRs) of ceftriaxone in paediatric patients.

Methods: We performed a systematic search in Medline, PubMed, Cochrane Central Register of Controlled Trials EMBASE, CINAHL, International Pharmaceutical Abstracts and bibliographies of relevant articles up to December 2018 for all types of studies that assessed the safety of ceftriaxone in paediatric patients aged $£ 18$ years.

Results: 112 studies met the inclusion criteria involving 5717 paediatric patients who received ceftfiaxone and reported 1136 ADRs. The most frequent ADRs reported in prospective studies were gastrointestinal (GI) disorders (37.4\%, $292 / 780$ ), followed by hepatobiliary disorders (24.6\%, 192/780). Serious ADRs leading to withdrawal or discontinuation of ceftriaxone were reported in 86 paediatric patients. Immune haemolytic anaemia $(34.9 \%, 30 / 86)$ and biliary pseudolithiasis $(26.7 \%, 23 / 86)$ were the two major causes. Haemolytic anaemia following intravenous ceftriaxone led to death in 11 children whose primary disease was sickle cell disease. Almost all bilary pseudolithiasis are reversible. However, the incidence was high affecting one in five paediatric patients $(20.7 \%)$.

Conclusions: G1-ADRs are the most common toxicity of ceftriaxone in paediatric patients. Immune haemolytic anaemia and biliary pseudolithiasis are the most serious ADRs and the major reasons for discontinuation of ceftriaxone. Immune haemolytic anaemia is more likely in children with sickle cell disease and may cause death. Ceftriaxone should be used with caution in children with sickle cell disease. 


\section{Evidence of the non-linear nature of skeletal maturation}

Boeyer ME, Leary EV, Sherwood RJ, et al

Arch Dis child 2020; 105: 631-638

doi: 10.1136/archdischild-2019-317652

Objective: The aim of this study was to assess longitudinal trajectories of skeletal maturation to determine if children exhibit periods of rapid maturation during normal childhood and adolescence.

Design: Retrospective longitudinal study. Patients: 345 participants, with an average of 25 assessments per participant, between 3 and 20 years of age from the Fels Longitudinal Study.

Main outcome measures: Chronological age (ie, timing) and rate (ie, tempo) of skeletal maturation, as assessed by the Fels Method, at each maturational milestone, as well as the duration of time spent between any two milestones, were calculated for each participant specific maturational trajectory and compared between three unique non-linear maturational trajectory types.

Results: More than $81 \%$ of participants exhibited a rapid period of skeletal maturation during childhood and/or adolescence, most of whom were characterised by a single maturational spurt during adolescence. Participants with only a single adolescent spurt in skeletal maturation reach adolescent onset and peak approximately 2.8 and 4.2 years earlier, respectively, in boys $(p<0$ 001) and girls $(p<0.001)$, than when compared with participants with both childhood and adolescent spurts. Differences in the timing and tempo of maturational milestones were driven primarily by trajectory type.

Conclusions: Rapid changes in skeletal maturation occur during normal childhood and/or adolescence, indicating the presence of a maturational spurt: a developmental phenomenon that has remained largely uncharacterised. This work highlights patterned changes in the timing, tempo and duration of longitudinal skeletal maturation while simultaneously shifting the paradigm that skeletal maturation progresses linearly.

\section{Minimal access retroperitoneal pancreatic necrosectomy for infected necrotizing pancreatitis: a multicentre study of a step-up approach}

Liu ZW, Yang SZ, Wang PF, et al

BJS 2020; 107: 1344-1353

DOI: $10.1002 /$ bjs. 11619

Background: Various minimally invasive approaches have been described for infected necrotizing pancreatities. This article describes a modified minimal access retroperitoneal pancreatic necrosectomy (MARPN) procedure assisted by gas insultation.

Methods: This retrospective, observational study documented patients who had undergone a stepup MARPN between 1 January 2010 and 31 December 2016. A minimum follow up of 1 year was required for inclusion. The step up approach involved percutaneous catheter drainage followed by the modified MARPN and necrosectomy. If more than one access site was needed it was categorized as complex MARPN.

Results: Of 212 patients with infected necrotizing pancreatitis, 164 (77.4 per cent) underwent a step up approach. The median number of percutaneous catheter drains and MARPN procedures was 3 (range 1-7) and 1 (1-6) respectively. Ninety patients (54.9 per cent) underwent complex MARPN. For residual necrosis after MARPN, three patients ( 18 per cent) underwent sinus tract gastroscopy, and 11 (6.7 per cent) had sinography combined with a tube change. However, operations in 13 patients (7.9 per cent) required conversion to open surgery. Postoperative complication developed in 103 patients (62.8 per cent). The mortality rate was 6.1 per cent (10 deaths).

Conclusion: A step up approach using a modified MARPN for infected necrotizing pancreatitis is a reasonable option.

\section{Rates of re-excision and conversion to mastectomy after breast conserving surgery with or without oncoplastic surgery: a nationwide population based study}

Heeg E, Jensen MB, Holmich IR, et al

BJS 2020; 107: 1762-1772

DOI : $10.1002 /$ bjs. 118

Background: There is no consensus regarding the impact of oncoplastic surgery (OPS) on rates of reexcision and conversion to mastectomy following breast conserving surgery (BCS). Here these two outcomes after BCS and OPS were compared in a nationwide population-based seecing.

Methods: In Denmark, all OPS is registered and categorized into volume displacement, volume reduction or volume replacement. Patients who underwent BCS or OPS between 2012 and 2019 were selected from the Danish Breast Cancer Group database. Multivariable analyses were performed to adjust for confounders, and propensity score matching to limit potential confounding by indication bias.

Results: A total of 13,185 patients (72.5 per cent) 
underwent BCS and 5003 (27.5 per cent) OPS. Volume displacement was used in 4171 patients (83.4 per cent, volume reduction in 679 (13.6 per cent) and volume replacement in 153 (3.1 per cent). Re-excision rates were 15.6 and 14.1 per cent after BCS and OPS respectively. After adjusting for confounders, patients were less likely to have a re-excision following OPS than BCS (odds ratio (OR) 0.80 , 95 per cent e.i. 0.72 to $0.88)$, specifically after volume displacement and reduction. The rate of conversion to mastectomy was similar after OPS and BCS (3.2 versus 3.7 per cent; $\mathrm{P}=0.105)$, but with a lower risk in adjusted analysis (OR 0.69, 0.58 to 0.84), specifically after volume displacement and reduction procedues. Findings were similar after propensity score matching.

Conclusion: A modest decrease in re-excision rate and less frequent conversion to mastectomy were observed after OPS compared with BCS.

\section{Magnesium sulphate for fetal neuroprotection at imminent risk for preterm delivery: a systematic review with meta analysis and trial sequential analysis}

Wolf HT, Huusom LD, Henriksen TB, et al

BJOG 2020; 127: 1180-1188

DOI: $10.1111 / 1471-0528,16238$

Background: Ordinary meta-analyses indicate that magnesium sulphate (MgS04) treatment in women at imminent risk for preterm delivery decreases the offspring's risk of cerebral palsy (CP). However, repetitive testing of cumulative data calls for statistical caution, e.g. by trial sequential analysis (TSA), for which there are previously insufficient samples to draw a firm conclusion. Recently, a randomised controlled trial (RCT) provided additional data that potentially increased the sample size such that a new TSA might detect a statistically significant effect.

Objectives: To assess the possible fetal neuroprotective effect of $\mathrm{MgS0} 4$ for women at imminent risk for preterm delivery in an updated systematic review with meta analysis and TSA.

Search strategy: We searched MEDLINE, Embase, Cochrane and Clinical Trials gov. on 8 October 2019. The search strategy clustered terms describing the MgSO4 intervention and preterm detivery.

Selection criteria: RCTs.

Data collection and analysis: Two reviewers extracted the data. Summary relative risks (R.Rs) and $95 \%$ confidence intervals (Cls) were calculated using fixed effects models. A TSA was applied to the primary outcome, CP. The quality of the evidence was aassessed using GRADE. The protocol was registered in PROSPERO (registration: CRD42019151441)

Main results: We identified six eligible trials (5917 women) MgSO4 intervention in women at imminent risk for preterm birth decreased the offspring's CP risk (meta-analysis RR 0.68, 95\% CI 0.54-0.85; TSA RR 0.69, 95\% CI 0.48-0.97).

Conclusions: This systematic review with meta analysis and TSA shows conclusively that MgS04, when given to women at imminent risk for preterm delivery, decreases the offspring's CP risk.

\section{Effect of hysterectomy on pain in women with endometriosis: a population based registry study}

Sandstrom A, Bixo $M$, Johaansson $M$, et al

BJOG 2020; 127: 1628-1635

DOI: $10.1111 / 1471-0578,16328$

Objective: To assess pain symptom before and after hysterectomy in women with endometriosis.

Design: A population based registry study.

Setting: Sweden.

Population: Women aged 18-45 years who underwent hysterectomy for endometriosis between 2010 and 2015 .

Methods: Pain symptoms before hysterectomy and 12 months after surgery were collected from the Swedish National Quality Register for Gynaecological Surgery (GynOp). Pain symptoms were also assessed by follow up surveys after a median follow up period of 63 months.

Main outcome measures: Pelvic or lower abdommal pain after hysterectomy.

Results: The study included 137 women. The proportion of women experiencing pain of any severity decreased by $28 \%$ after hysterectomy $(\mathrm{P}<$ $0.001)$. The proportion of women with severe pain symptoms decreased by $76 \%$ after hysterectomy $(\mathrm{P}<0.001)$. The majority of women $(84 \%)$ were satisfied with the surgical result. Presence of severe pain symptoms after the hysterectomy was associated with less satisfaction $(\mathrm{P}<0.001)$. Pain symptoms after surgery, patient satisfaction and the patient's perceived improvement were not significantly different between women whose ovarian tissue was preserved and women who underwent bilateral oophorectomy.

Conclusions: We observed a significant, longlasting reduction in pain symptoms after hysterectomy among women with endometriosis. Hysterectomy, with the possibility of ovarian preservation, may be a valuable option for women with endometriosis who suffer from severe pain symptoms. 